\title{
Atomic-scale thermal behavior of nanoimprinted 0.3-nm-high step patterns on PMMA polymer sheets
}

\author{
Goon Tan ${ }^{1}$, Yasuhisa Nozawa ${ }^{1}$, Tomoyuki Funabasama ${ }^{1}$, Koji Koyama ${ }^{2}$, Masahiro Mita ${ }^{3}$, Satoru Kaneko ${ }^{1,4}$, \\ Motonori Komura $^{5}$, Akifumi Matsuda ${ }^{1}$ and Mamoru Yoshimoto ${ }^{1}$
}

Polymer Journal (2016) 48, 225-227; doi:10.1038/pj.2015.99; published online 28 October 2015

\section{INTRODUCTION}

The resolution limit of patterning polymer surfaces has attracted much attention from both practical and academic viewpoints. The nanoimprint process is one of the most promising techniques for simple, low-cost and high-throughput nanopatterning. ${ }^{1}$ To date, novel feature sizes $<5 \mathrm{~nm}$ have been demonstrated. ${ }^{2,3}$ There are several types of nanoimprinting. One is thermal nanoimprinting, which is applied to thermoplastic polymers such as poly(methyl methacrylate) (PMMA) and polystyrene (PS). ${ }^{4,5}$ Recently, we reported subnanometer-scale surface patterning on soda-lime silicate glasses and PMMA polymer sheets by the thermal nanoimprint technique, ${ }^{6,7}$ in which we applied a self-organized nanopattern mold of atomically stepped sapphire $\left(\alpha-\mathrm{Al}_{2} \mathrm{O}_{3}\right.$ single crystal) as the imprint template. ${ }^{8}$ The atomically stepped sapphire substrates were also used for growing high quality thin films at low temperatures ${ }^{9,10}$ and as sample stages for observing the steric shape of organic molecules adhered to the surfaces by atomic force microscopy (AFM). ${ }^{11,12}$ These imprinted PMMA surfaces exhibited regularly arrayed atomic stairs with $\sim 0.3-\mathrm{nm}$-high steps, reflecting the sapphire template's pattern. ${ }^{7}$ Further atomic-scale investigations into the effect of imprinting conditions such as press temperature on transcription are thought to be necessary for the development of atomic-scale polymer surface engineering.

In addition, thermal deformation and relaxation of patterned polymer surfaces are intriguing in the light of both scientific and technological perspectives. ${ }^{13-15}$ It is important to examine the thermal stability of the atomically stepped pattern formed on a PMMA surface at the atomic scale to inform applications such as the use of substrates for observing macromolecules or growing functional thin films. In the present work, we examined the effect of imprinting temperature on the transcription of an atomic step pattern. We also observed atomicscale thermal changes in the atomically stepped pattern on the PMMA surface using high temperature in situ AFM. ${ }^{16,17}$ An in situ AFM apparatus equipped with a sample heating stage enabled us to observe the thermal behavior of the polymer surface directly at a high temperature.

\section{EXPERIMENTAL PROCEDURE}

\section{Nanoimprint procedure}

An atomically stepped sapphire mold was obtained by annealing a mirrorpolished sapphire (10-12; r-plane) wafer (Namiki Precision Jewel, Tokyo, Japan) at $1200^{\circ} \mathrm{C}$ for $3 \mathrm{~h}$ in air. ${ }^{6}$ PMMA sheets (Mitsubishi Rayon, Acrylite S) with $\sim 2$-mm thickness were used for thermal nanoimprinting. The average molecular weight $(\mathrm{Mn})$ of this PMMA is $\sim 10^{6} \mathrm{~g} \mathrm{~mol}^{-1}$. Nanoimprinting was performed on a nanoimprint machine (X300, SCIVAX, Kanagawa, Japan) that consisted of two heating stages. The sample sizes of the sapphire mold and PMMA sheet were each $1.0 \times 1.0 \mathrm{~cm}^{2}$. The sapphire mold was placed on the lower heating stage and the PMMA sheet was placed on the stepped surface of the sapphire mold. The imprinting temperature was controlled between 80 and $140^{\circ} \mathrm{C}$. When the upper and lower stages achieved the set temperature, we applied a pressure not exceeding $0.2 \mathrm{MPa}$ for $300 \mathrm{~s}$ in air. We subsequently cooled the two stages to $30^{\circ} \mathrm{C}$ and then released the pressure.

\section{Characterization}

Differential scanning calorimeter analysis was performed using a DSC calorimeter (DSC7020, Hitachi High-Tech Science, Tokyo, Japan) to estimate the glass transition temperature of the PMMA sheets used in this study. PMMA powder $\left(10 \mathrm{mg}\right.$ in an aluminum pan) was heated from 30 to $180^{\circ} \mathrm{C}$ and cooled back to $30^{\circ} \mathrm{C}$; we repeated this heat cycle several times to obtain precise data. The heating rate was set at $10^{\circ} \mathrm{C} \mathrm{min}-1$ and alpha-alumina powder was used as a reference substance.

The surface morphology and roughness of imprinted PMMA sheets were characterized in air by atomic force microscopy (AFM; Nanocute, Hitachi High-Tech Science) using microfabricated Si cantilevers (SI-DF40P2, Hitachi High-Tech Science) in dynamic force mode. To estimate the influence of imprinting temperature on the transcription, we characterized the average root mean square (RMS) roughness on the step terraces of patterned PMMA imprinted at various temperatures. Furthermore, atomic-scale deformation behavior during thermal annealing from room temperature to $140^{\circ} \mathrm{C}$ was examined by in situ AFM (NanoScope IV, Multimode, Bruker) using Si cantilevers (Tap150, Bruker, Tokyo, Japan).

${ }^{1}$ Department of Innovative and Engineered Materials, Tokyo Institute of Technology, Yokohama, Japan; ${ }^{2}$ Namiki Precision Jewel Company Limited, Tokyo, Japan; ${ }^{3}$ Kyodo International Incorporated, Kawasaki, Japan; ${ }^{4}$ Kanagawa Industrial Technology Research Institute, Kanagawa, Japan and ${ }^{5}$ Department of Electrical and Electronics Engineering, Numazu National College of Technology, Shizuoka, Japan

Correspondence: Professor M Yoshimoto, Department of Innovative and Engineered Materials, Tokyo Institute of Technology, 4259 J3-16, Nagatsuta, Midori, Yokohama 226-8502, Japan.

E-mail: yoshimoto.m.aa@m.titech.ac.jp

Received 20 May 2015; revised 27 August 2015; accepted 27 August 2015; published online 28 October 2015 


\section{RESULTS AND DISCUSSION}

The differential scanning calorimeter analysis (the third run of heat cycle) indicated that the bulk glass transition temperature $\left(T_{\mathrm{g}}\right)$ of PMMA is $\sim 105^{\circ} \mathrm{C}$. We then investigated the transcription of an atomically stepped pattern onto PMMA under various imprinting temperatures near its $T_{\mathrm{g}}$, which is related to structural relaxation of glassy polymers. When the imprinting temperature was $80^{\circ} \mathrm{C}$, we observed no pattern and the surface remained rough and similar to that of the untreated PMMA surface (Figure 1a). When the temperature was increased to $90^{\circ} \mathrm{C}$, we identified step shapes on some areas. However, the surface was still rough and exhibited many large scratches as shown in Figure 1b. After imprinting at a
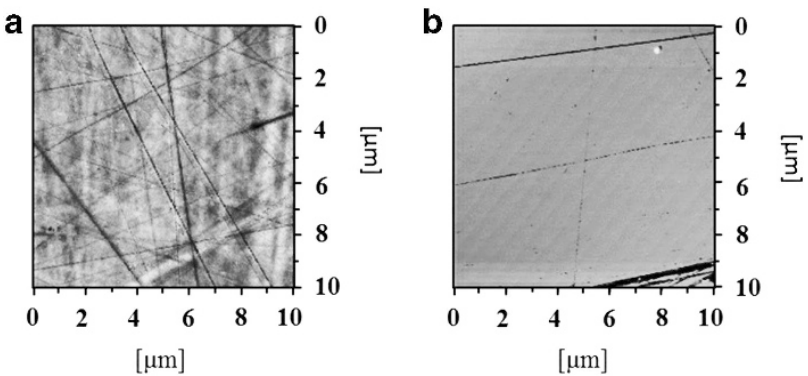

Figure 1 Atomic force microscopy (AFM) images $\left(10 \times 10 \mu \mathrm{m}^{2}\right)$ of (a) an untreated poly(methyl methacrylate) (PMMA) surface and (b) the PMMA surface imprinted at $90^{\circ} \mathrm{C}$. A full color version of this figure is available at Polymer Journal online. a
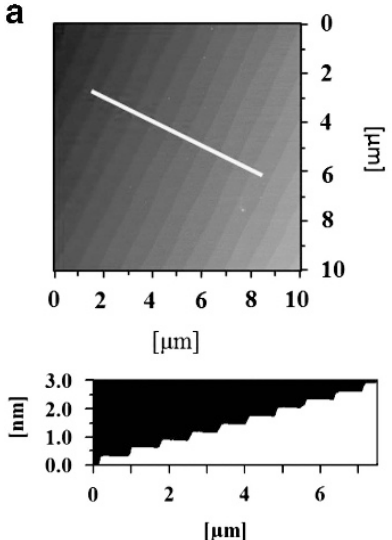

b
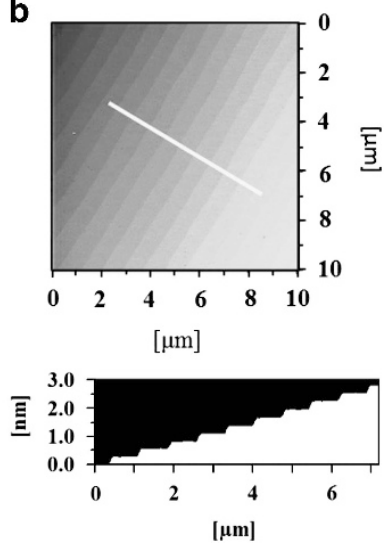

Figure 2 Tilted atomic force microscopy (AFM) images $\left(10 \times 10 \mu \mathrm{m}^{2}\right)$ and cross-sectional profiles along the white lines of (a) the atomically stepped sapphire mold and (b) the poly(methyl methacrylate) (PMMA) surface imprinted at $120^{\circ} \mathrm{C}$. A full color version of this figure is available at Polymer Journal online. temperature above $T_{\mathrm{g}}\left(105^{\circ} \mathrm{C}\right)$, we observed large-area transcription of the atomically stepped pattern onto the PMMA surface. The atomic-scale pattern could be transferred at very small pressure $(<0.2 \mathrm{MPa})$ in our study. In contrast, some previous papers reported that transfer of nanoscale structures onto PMMA surfaces generally requires a pressure exceeding several $\mathrm{MPa}^{4,5}$ To examine the step height precisely, we used the area tilt function in the AFM software to level and incline the surface images.

Figure 2 shows the tilted AFM images and the cross-sectional profiles of (Figure 2a) the sapphire mold and (Figure 2b) the PMMA sheet imprinted at $120^{\circ} \mathrm{C}$. As shown in Figure 2a, the sapphire mold exhibits regularly arranged atomic steps with a terrace width of $700-800 \mathrm{~nm}$ and a uniform height of $0.34( \pm 0.01) \mathrm{nm}$. The step terrace width and height of imprinted PMMA as shown in Figure $2 b$ are $700-800 \mathrm{~nm}$ and $0.29( \pm 0.01) \mathrm{nm}$, respectively, which are similar to those of the sapphire mold. The decrease in atomic step height for the imprinted PMMA surface might be caused partly by thermal shrinkage during the cooling process or by viscoelastic relaxation of macromolecules as a result of the stress induced near the step edges in the molding process.

Figure 3 shows the average RMS roughness and deviation of the atomic terraces for $50 \times 50 \mathrm{~nm}^{2}$ areas between steps on the sapphire mold and PMMA sheets imprinted at various temperatures. The deviation of each roughness value in Figure 3 was estimated from ten areas of $50 \times 50 \mathrm{~nm}^{2}$ on the atomic terraces. Surface roughness changed little between 90 and $105^{\circ} \mathrm{C}$ (below $T_{\mathrm{g}}$ ). When increasing the temperature from 110 to $120^{\circ} \mathrm{C}$ (above $T_{\mathrm{g}}$ ), it was observed that the roughness of the terraces became smaller and closer to that of the sapphire surface. Flattening of the terraces, as shown in Figure 3, was attributed to an increase in the fluidity of the polymer surface at

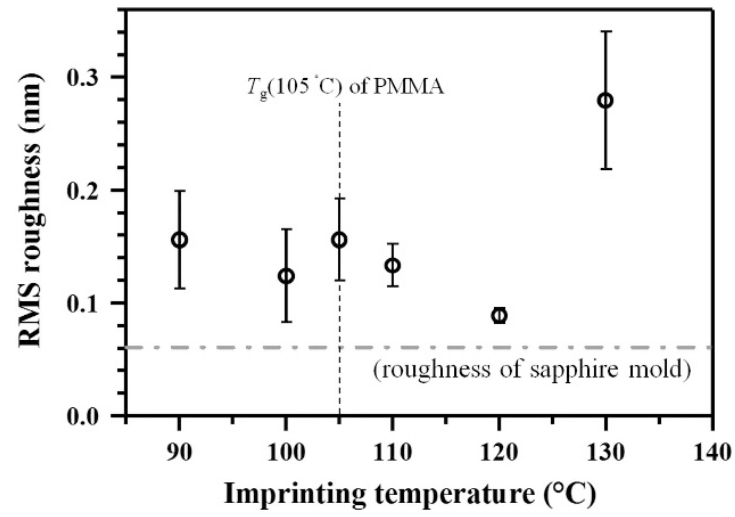

Figure 3 Average root mean square (RMS) roughness of atomic terraces between steps on the imprinted poly(methyl methacrylate) (PMMA) with various imprinting temperatures. A full color version of this figure is available at Polymer Journal online.
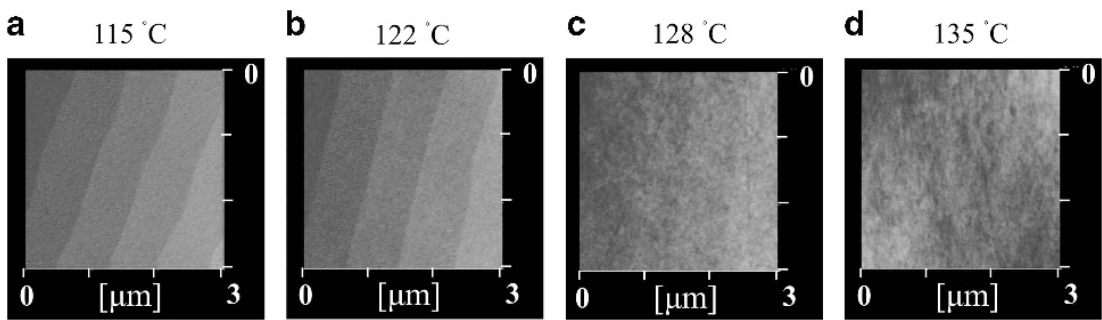

Figure 4 In situ atomic force microscopy (AFM) images $\left(3 \times 3 \mu \mathrm{m}^{2}\right)$ of the atomically stepped poly(methyl methacrylate) (PMMA) observed at (a) 115 , (b) 122 , (c) 128 and (d) $135^{\circ} \mathrm{C}$. A full color version of this figure is available at Polymer Journal online. 
higher press temperatures. However, the roughness was found to increase again at $130{ }^{\circ} \mathrm{C}$. This increase of terrace roughness might be caused by partial adhesion of the polymer onto the mold surface and lateral shifting induced by the thermal mismatch of the mold and the polymer during higher-temperature pressing. At $140{ }^{\circ} \mathrm{C}$, the mold and the PMMA sheet adhered strongly and it was difficult to release them after imprinting. We also performed supplemental measurements to estimate the temperature at the interface between sapphire and PMMA using a thermocouple. The thin thermal sensor head was inserted between the sapphire and PMMA and the usual nanoimprint process was performed. During imprinting at $140^{\circ} \mathrm{C}$, the sensor displayed a temperature of $142-143^{\circ} \mathrm{C}$, indicating that the interfacial temperature of the PMMA was close to that of the heater. Next, we investigated the atomic-scale thermal change of the 0.3-nm-high stepped pattern on the PMMA surface during heating by applying the in situ AFM system. Figures $4 \mathrm{a}$ and $\mathrm{b}$ reveal that the atomically stepped pattern was unchanged up to $122^{\circ} \mathrm{C}$, which is greater than the $T_{\mathrm{g}}$ of $105^{\circ} \mathrm{C}$. Figures $4 \mathrm{c}$ and $\mathrm{d}$ indicate that the step pattern seemed to fade away partially at $128^{\circ} \mathrm{C}$ and completely disappeared after the AFM stage was heated to $135^{\circ} \mathrm{C}$. Thus, the PMMA surface became rougher and exhibited more scratches at higher temperatures, suggesting that thermal deformation of the atomic pattern occurred mainly by viscous flow characteristic of amorphous materials. Tsui and co-workers have reported that the mobility at the polymer surface layer is generally enhanced in comparison with the bulk, ${ }^{18}$ although there are controversial arguments against mobility-enhanced surface dynamics. Our results of in situ AFM observation indicate that the atomic-scale pattern on the PMMA surface was stable above the $T_{\mathrm{g}}$ of $105^{\circ} \mathrm{C}$. Although it might be challenging to estimate the $T_{\mathrm{g}}$ value near the topmost surface of the PMMA, special care was taken in the area of temperature control during in situ AFM observation. For instance, the temperature was set for both the sample stage and the AFM cantilever. After reaching the same temperature, the AFM system temperature was held for $>5 \mathrm{~min}$. Then, we started in situ observation. Further studies are necessary to clarify the detailed mechanism of thermal deformation in terms of the dynamic molecular motion near the 0.3-nm-high atomic steps of an imprinted PMMA surface.

In summary, we examined the imprinting temperature and thermal deformation of $0.3-\mathrm{nm}$-high step patterns formed on a PMMA surface. We obtained large-area transcription and flat step terraces under the condition of $\sim 0.2 \mathrm{MPa}$ load for $300 \mathrm{~s}$ at $120^{\circ} \mathrm{C}$. To study the thermal deformation of an atomically stepped pattern during heating, we used in situ AFM with a stage heater. We found that the atomically stepped pattern was stable at temperatures greater than its $T_{\mathrm{g}}$ of $105^{\circ} \mathrm{C}$.

\section{CONFLICT OF INTEREST}

The authors declare no conflict of interest.

\section{ACKNOWLEDGEMENTS}

This study was partly supported by JSPS KAKENHI and New Energy and Industrial Technology Development Organization (NEDO).

1 Chou, S. Y, Krauss, P. R. \& Renstrom, P. J. Imprint of sub-25 nm vias and trenches in polymers. Appl. Phys. Lett. 67, 3114-3116 (1995).

2 Austin, M. D., Ge, H., Wu, W., Li, M., Yu, Z., Wasserman, D., Lyon, S. A. \& Chou, S. Y. Fabrication of $5 \mathrm{~nm}$ linewidth and $14 \mathrm{~nm}$ pitch features by nanoimprint lithography. Appl. Phys. Lett. 84, 5299-5301 (2004).

3 Hua, F., Gaur, A., Sun, Y., Word, M., Jin, N., Adesida, I., Shim, M., Shim, A. \& Rogers, J. A. Processing dependent behavior of soft imprint lithography on the 1-10-nm scale. IEEE Trans. Nanotechnol. 5, 301-308 (2006).

4 Chou, S. Y., Krauss, P. R. \& Renstrom, P. J. Imprint lithography with 25-nanometer resolution. Science 272, 85-87 (1996).

5 Hirai, Y., Fujiwara, M., Okuno, T. \& Tanaka, Y. Study of the resist deformation in nanoimprint lithography. J. Vac. Sci. Technol. B 19, 2811-2815 (2001).

6 Akita, Y., Watanabe, T., Hara, W., Matsuda, A. \& Yoshimoto, M. Atomically stepped glass surface formed by nanoimprint. Jpn J. Appl. Phys. 46, L342-L344 (2007).

7 Tan, G., Inoue, N., Funabasama, T., Mita, M., Okuda, N., Mori, J., Koyama, K., Kaneko, S., Nakagawa, M., Matsuda, A. \& Yoshimoto, M. Formation of 0.3-nm-high stepped polymer surface by thermal nanoimprinting. Appl. Phys. Express 7, 3 (2014).

8 Yoshimoto, M., Maeda, T., Ohnishi, T., Koinuma, H., Ishiyama, O., Shinohara, M., Kubo, M., Miura, R. \& Miyamoto, A. Atomic-scale formation of ultrasmooth surfaces on sapphire substrates for high-quality thin-film fabrication. Appl. Phys. Lett. 67, 2615-2617 (1995).

9 Yoshimoto, M. Yoshida, K. Maruta, H., Hishitani, Y. Koinuma, H., Nishio, S. Kakihana, M. \& Tachibana, T. Epitaxial diamond growth on sapphire in an oxidizing environment. Nature 399, 340-342 (1999).

10 Sasaki, A., Hara, W., Matsuda, A., Tateda, N., Otaka, S., Akiba, S., Saito, K., Yodo, T. \& Yoshimoto, M. Buffer-enhanced room-temperature growth and characterization of epitaxial ZnO thin films. Appl. Phys. Lett. 86, 3 (2005).

11 Ebihara, K., Koshihara, S., Yoshimoto, M., Maeda, T., Ohnishi, T., Koinuma, H. \& Fujiki, M. Direct observation of helical polysilane nanostructures by atomic force microscopy. Jpn J. Appl. Phys. 36, L1211-L1213 (1997).

12 Yoshida, K., Yoshimoto, M., Sasaki, K. \& Ohnishi, T. Fabrication of a new substrate for atomic force microscopic observation of DNA molecules from an ultrasmooth sapphire plate. Biophys. J. 74, 1654-1657 (1998).

13 Jones, R. L., Hu, T., Soles, C. L., Lin, E. K., Reano, R. M., Pang, S. W. \& Casa, D. M. Real-time shape evolution of nanoimprinted polymer structures during thermal annealing. Nano Lett. 6, 1723-1728 (2006)

14 Ding, Y., Ro, H. W., Alvine, K. J., Okerberg, B. C., Zhou, J., Douglas, J. F., Karim, A. \& Soles, C. L. Nanoimprint lithography and the role of viscoelasticity in the generation of residual stress in model polystyrene patterns. Adv. Funct. Mater. 18, 1854-1862 (2008).

15 Mundra, M. K., Donthu, S. K., Dravid, V. P. \& Torkelson, J. M. Effect of spatial confinement on the glass-transition temperature of patterned polymer nanostructures. Nano Lett. 7, 713-718 (2007).

16 Takanashi, Y. \& Kumaki, J. Significant melting point depression of two-dimensional folded-chain crystals of isotactic poly(methyl methacrylate)s observed by high-resolution in situ atomic force microscopy. J Phys Chem B. 117, 5594-5605 (2013).

17 Komura, M., Komiyama, H., Nagai, K. \& lyoda, T. Direct observation of faceted grain growth of hexagonal cylinder domains in a side chain liquid crystalline block copolymer matrix. Macromolecules 46, 9013-9020 (2013).

18 Yang, Z., Fujii, Y., Lee, F. K., Lam, C. H. \& Tsui, O. K. C. Glass transition dynamics and surface layer mobility in unentangled polystyrene films. Science 328, 1676-1679 (2010). 